\title{
La Dahira de Mame Diarra en la diáspora: ¿un desafío al patriarcado murid?*
}

\author{
The Mame Diarra dahira in the diaspora: \\ challenging Murid patriarchy?
}

\author{
Ester Massó Guijarro \\ Departamento de Antropología Social \\ Facultad de Filosofía y Letras. Universidad de Granada
}

\section{RESUMEN}

La dahira (agrupación de fieles en torno a un líder religioso, con cometidos colectivos espirituales y materiales) constituye el modo organizativo por antonomasia de la cofradía Muridiyya, tanto en Senegal como en su intensa diáspora migratoria. Las dahiras exclusivamente femeninas, frente a las masculinas o mixtas, se sostienen sobre la base del culto a Mame Diarra, figura inmensamente respetada como madre de Ahmadou Bamba, fundador del muridismo. Tales dahiras funcionan de modo autónomo e independiente en todos los ámbitos: gestión económica, organización, liderazgo, etc., movilizando gran cantidad de recursos humanos y económicos dentro y fuera de Senegal. Este artículo revisa la teoría más relevante sobre las dahiras, en primer lugar, y las dahiras femeninas desde una perspectiva de género, en segundo lugar, para abordar después el estudio de caso de la dahira de Mame Diarra en Madrid y su significación en el contexto de la comunidad murid en España a fin de analizar si, efectivamente, la experiencia del muridismo para las mujeres a través de las dahiras conlleva el grado de autonomía y agencia que algunos estudios defienden.

Palabras clave: Mujeres murid, Diáspora migratoria, Senegal, Dahiras femeninas, Mame Diarra.

\section{SUMMARY}

The dahira (a group of believers around a religious leader, with both espiritual and material collective committments) is the quintessential organizational mode of the brotherhood Muridiyya, both in Senegal and in its intense migrant diaspora. The exclusively female dahiras, against male or mixed, are held on the basis of the cult of Mame Diarra, a hugely respected figure as the mother of Ahmadou Bamba, founder of Muridism. Such dahiras operate autonomously and inde-

\footnotetext{
* Este trabajo se ha elaborado en el marco del proyecto de investigación "Integración, participación y justicia social" (FFI2009-07056), financiado por el Ministerio de Ciencia e Innovación y dirigido por Juan Carlos Velasco Arroyo (IP). Una versión preliminar y reducida del presente artículo se presentó a modo de comunicación en el I Congreso Internacional FEMIGRA: Feminismo, migración e intervención social", (Barcelona, 9-11 febrero 2012). Agradezco a José Ángel Martínez Casares, Iria Vázquez Silva y Juan Antonio Estrada Díaz la lectura del manuscrito original y los valiosos comentarios al mismo. Asimismo, agradezco a la comunidad murid en Madrid su imprescindible y amable cooperación para el desarrollo de mi trabajo; y, por supuesto, su amistad.
} 
pendently at all levels: economic management, organization, leadership, etc., mobilizing large amounts of human and financial resources within and outside Senegal. This article reviews the relevant theory on dahiras firstly, and female dahiras from a gender perspective, secondly, to address after the case study of Mame Diarra dahira in Madrid and its significance in the Murid community context in Spain, to analyze whether Muridism experience for women through dahiras involves the degree of autonomy and agency that some studies claim.

Key words: Murid Women, Migrant Diaspora, Senegal, Female Dahiras, Mame Diarra.

\section{INTRODUCCIÓN: PLANTEAMIENTO Y OBJETIVOS}

"[...] las diversas sendas de la religiosidad musulmana del África negra y malgache, una realidad polimorfa, creativa y estructurante en muchos países del continente ${ }^{1}{ }_{\text {" }}$ (Iniesta Vernet 2009: 12).

La migración senegalesa es la más numerosa en España de entre las diversas procedencias subsaharianas (Jabardo Velasco 2006; Vázquez Silva 2011²). Asimismo, es la que presenta una actividad asociativa más intensa entre ellas, tanto en la forma de un asociacionismo que podríamos denominar "a la occidental", como, y muy especialmente, a través de formas asociativas autóctonas - a menudo ni siquiera registradas oficialmente como tales-, la mayoría enraizadas en la práctica religiosa del muridismo (Sow 2004; Jabardo Velasco 2006; Crespo Ubero 2006; Moreno Maestro 20073).

La cofradía sufí-musulmana ${ }^{4}$ Muridiyya $^{5}$ (la más numerosa en la diáspora migratoria frente a otras cofradías análogas) opera como red y como colectivo migratorio donde

${ }^{1}$ La especificidad del llamado "islam negro" ha sido abordada por numerosos autores. Ver también Iniesta Vernet (1998), Mazrui (2000), Salter (2000), Piga (2003), Cruise O’Brien (2003), Brenner (2005) y Coulon (2009).

${ }^{2}$ La medición de la intensidad y el número de personas en las migraciones transnacionales no se presenta exenta de dificultad metodológica. El reciente trabajo de Vázquez Silva (2011) realiza un análisis sobre los flujos migratorios Senegal-España desde la superación del nacionalismo metodológico, denunciando la mutilación del objeto de estudio que suponía aquél: "En este caso la mutilación resulta especialmente grave, dado que la bibliografía especializada presenta la reciente migración internacional senegalesa, principalmente el modelo módou-módou, como un paradigma de las migraciones transnacionales" (Vázquez Silva 2011: 4).

3 Para contrastar el cercano caso del asociacionismo senegalés en Italia, ver los estudios de Bruno Riccio (2011).

4 Puede parecer ocioso o redundante matizar la categoría "Sufí" con "musulmana" y, sin embargo, no lo es. Pese a la generalizada creencia de que la corriente sufí es per se musulmana, y si bien es cierto que la vía por la cual el sufismo se extendió mayormente fue en su confluencia con el islam, el origen del sufismo es anterior al surgimiento de aquél (sus orígenes no han podido ser averiguados ni fechados, explica Shah 2003), y la confluencia entre sufismo e islam es más bien histórica y debido al carácter del culto musulmán, que se compadeció especialmente bien con las características del sufismo: "Y si llaman al Islam 'caparazón' del sufismo es porque consideran al sufismo como la enseñanza secreta contenida en todas las religiones" (Shah 2003: 9). Ver también Skali (1993: 8), sobre la relación íntima pero no necesaria entre sufismo e islam.

${ }^{5}$ He optado por una transcripción concreta frente a las variadas que existen sobre los términos "murid" (o "murides"), "Muridiyya", "marabout", etc. El criterio que me ha guiado ha sido, fundamentalmente, la sencillez en la transcripción y la mayor semejanza a la pronunciación hispana (dado que tratamos de términos que suelen proceder del wolof oral —a veces del árabe-, existen 
se arraciman las prácticas de negociación identitaria de toda índole en la migración senegalesa (Guèye 2008: 63). Las dahiras (una forma específica de asociaciones) constituyen las agrupaciones que funcionan en la diáspora para redistribuir, atender, organizar, asistir, facilitar, festejar, etc. Casi siempre establecidas en torno a la figura de un líder espiritual masculino (marabout o sheikh ${ }^{6}$ ), no escapan de la estructura básica patriarcal, tanto del muridismo como de las diferentes culturas senegalesas (Bop 2005).

En mi experiencia de trabajo de campo con senegaleses murid en Madrid, me interesa especialmente el ejercicio de la ciudadanía a través de los movimientos colectivos y, por tanto, de las diferentes dahiras; la masculina (Dahira Serigne Fallou-Touba ${ }^{7}$ Madrid) y, especialmente para este trabajo, la dahira femenina, Mame Diarra "Mouhadjirat". Como es habitual en numerosos sistemas espirituales y de creencias, el lugar de la mujer en el muridismo no es equiparable, en derechos y deberes, al del hombre, y existe un aparente sometimiento que se enmarca en valores culturales más amplios (Creevey 1991). Sin embargo, también se ha reseñado que las mujeres murid gozan de una agencia independiente (económica, pero no solo) inusual en comparación con otros grupos islámicos (Coulon 1988; Coulon y Reveyrand Coulon 1990; Evers Rosander 1996, 1997a, 1997b; Buggenhagen 2001, 2009; Diouf y Leichtman 2009). Influye en ello de modo crucial el culto a la maternidad (Evers Rosander 2003a, 2003b; Roberts, Nooter y Armenian 2003a y 2003b), con profundas raíces en las sociedades donde el muridismo nació, y que confiere un peso específico a la mujer-madre, su control social, sus deberes y sus derechos. Hechos como la existencia de marabout mujeres (Gemmeke 2009), o la intensa capacidad de agencia de las mujeres murid en sus dahiras, abren vetas en un muridismo que aparenta ser, por ende, esencialmente patriarcal.

El estudio de caso que operará aquí como pretexto o ejemplo reflexivo es, a la sazón, el establecimiento de la dahira de Mame Diarra en Madrid: una asociación exclusivamente femenina, de y para la agencia de mujeres en la diáspora, que desafía muchas asunciones habituales sobre el islam (o cualquier religión monoteísta mayoritaria) en general y el muridismo (o cualquier versión sufí del islam) en particular. Interesa analizar, por un lado, cómo la figura de la dahira femenina en sí (dentro y fuera de Senegal) constituye una esfera de agencia autónoma para las mujeres; por otro lado, cómo en la diáspora pueda agudizarse o no el elemento de emancipación que supone para las mujeres el ejercicio de la autonomía decisoria a través de la dahira (teniendo en cuenta el contexto occidental de familia nuclear donde cambian radicalmente las esferas en las que las mujeres senegalesas murid suelen operar).

Este artículo se articula en tres partes fundamentales. En un primer momento (epígrafe 2) trataremos de algunos de los rasgos fundamentales de las hermandades

múltiples transcripciones aceptables y frecuentes en los textos científicos). Asimismo, evito el uso de cursivas en estas y otras palabras extranjeras para no incomodar la lectura, y ya que son abundantes en el presente artículo.

${ }^{6}$ Apelan a un líder religioso y pueden considerarse sinónimos. Para una etimología interesante del término "marabout", ver Gemmeke (2009: 129 y ss). Para una aproximación a las funciones y caracteres de estos jefes religiosos, ver Lake (1997), Beck (2001) y Schmizt (1983).

7 Touba, hoy la segunda ciudad senegalesa después de Dakar, fue construida en el lugar de nacimiento de Ahmadou Bamba. Se ha erigido como ciudad santa de la cofradía, capital del muridismo (Guèye 2008) y lugar de obligado peregrinaje, incluso frente a Meca. 
(dahiras) en la diáspora migratoria murid, y por qué considero que en ellas se desempeña el ejercicio de la ciudadanía concebido de otro modo. En un segundo momento (epígrafe 3) se abordará más específicamente el muridismo desde una perspectiva de género $^{8}$, con especial énfasis en la existencia de las dahiras femeninas y la configuración de una agencia de actuación independiente. En un tercer momento (epígrafe 4) se tratará, como estudio de caso, de la dahira femenina establecida en Madrid. En nuestras reflexiones conclusivas (epígrafe 5) trataremos de vincular los datos teóricos con los hallazgos empíricos para ofrecer una visión informada sobre la cuestión tratada y algunas posibles respuestas a la interrogante del título de este trabajo.

La metodología empleada para la investigación empírica base del presente texto es el trabajo de campo antropológico (observación participante y entrevistas abiertas y semiestructuradas en profundidad), comenzando en enero de 2011 y en curso hasta hoy. En la investigación se trata con una muestra intencional de población diversificada en función de la nacionalidad (senegalesa), la pertenencia a la cofradía murid, el género (mujeres, hombres) y la edad (franja 20-30 años/ 30-50 años aprox.). La observación se ha desarrollado fundamentalmente en el barrio de Lavapiés y en la Casa de Serigne Touba a las afueras de Madrid.

\section{LA CIUDADANÍA A TRAVÉS DE LA DAHIRA: EPISTEMOLOGÍA Y COSMOLOGÍA MURID}

Dahira are founded wherever a Murid community exists (Babou 2002: 154).

La Muridiyya constituye una cofradía sufí que presenta interesantes peculiaridades en relación a otras cofradías análogas. Es la única nacida específicamente en Senegal. Fue fundada en el siglo XIX por el sheikh Ahmadou Bamba (también llamado Serigne 9 Touba; Senegal, 1853-1927) como esqueje de la Qadriyya ${ }^{10}$. Desde el principio apareció ligada a una ética del trabajo como vía de purificación espiritual (frente a una dedicación mayor al rezo, por ejemplo), a la contestación colonial (mixturada con la alianza, como solía suceder), a la migración (primero del ámbito rural al urbano y después al transnacional ${ }^{11}$ ) (Tidiane 1967; Copans 1988, 2000; Cruise O'Brien 2003: 64 y ss.) y a una tremenda adaptación evolutiva en los contextos sociales cambiantes, lo que resulta de gran utilidad en la diáspora.

El muridismo posee, como hecho complejo, numerosos ángulos y perspectivas. Se

\footnotetext{
${ }^{8}$ Con perspectiva de género no me refiero simplemente a un estudio sobre las mujeres, sino a un énfasis especial en las relaciones entre diferentes géneros, cómo se establecen sus diferencias o vecindades, y cómo el género funciona en tales relaciones y las realidades sociales implicadas, para significar y devenir un eje de comprensión (y vertebrador) de praxis colectivas.

9 "Serigne" es la palabra en wolof para designar al marabout o sheikh (Gemmeke 2009).

${ }^{10}$ Existen tres cofradías sufíes principales en Senegal: la Qadriyya, la Tijaniyya y la Muridiyya, seguidas de lejos por la Layenne.

${ }^{11}$ La migración murid del campo a la ciudad (rural-urbana) devino crucial para realzar y solidificar la eficacia de la dahira en su papel de inserción y cohesión social ante el nuevo escenario, especialmente en la gran urbe de Dakar. El comercio adquiere una importancia novedosa y se desarrollan pautas de solidaridad inéditas y específicas (comunicación personal de Iria Vázquez Silva, 16/02/2012).
} 
habla de él como religión, pero también como ideología (Cruise O’Brien 1988: 144) ${ }^{12}$. Constituye una cofradía musulmana sufí, que, sin embargo, presenta prácticas que la distancian del sufismo clásico y, por ende, del islamismo más ortodoxo de las escuelas coránicas (Gómez-Pérez 1998: 38; Costa Dias 2009). Así, en el seno de la heterodoxia que ya el sufismo presenta dentro del islam, la versión murid contiene especificidades culturales que la desmarcan también de un sufismo ortodoxo (M'Backé 2005; Samson 2009). Su arraigo en Senegal, su tradición migratoria, su intenso misticismo combinado con el amor al trabajo manual en el seno del modo capitalista de producción, su proyección hoy transnacional ${ }^{13}$ (Kane 2011) o la obediencia a la autoridad (ligada también a lo político, a menudo ${ }^{14}$ ) (Samson 2000; Loimeier 2009) de los sheikhs son algunos de los elementos claves de su idiosincrasia ${ }^{15}$.

Se ha afirmado (Coulon 1981) que en Senegal se es antes talibe, discípulo de un marabout, que ciudadano de un Estado. Esta afirmación de los años ochenta ha sido revisada por numerosos estudios más recientes y, sin embargo, continúa siendo cierta (parcialmente al menos, como son todas las verdades científicas). Senegal continúa siendo hoy, como fue llamado entonces, el paraíso de las cofradías, y la obediencia a un jefe religioso continúa, tanto en Senegal como en su intensa diáspora migratoria, gozando de buena salud entre los talibe. La obediencia a un líder espiritual, sumada al rechazo de autoridades más mundanas, constituye un rasgo claramente sufí del muridismo. Tal sumisión se ve reforzada por la concepción del carisma poseído por los marabouts (Cruise O'Brien 1969, 1970, 1988) y por la importancia que posee en el sufismo el aspecto de enseñanza e iniciación espiritual del marabout hacia el talibe (Babou 2003; Skali 2006: 28 y ss.). Este sistema de fidelidad es a menudo juzgado por ciertos académicos como un sistema de explotación capitalista (Cruise O'Brien 1969), si bien es evidente que los talibe admiten y fomentan este vínculo de forma autónoma y obtienen de él claros beneficios, moviéndose en lógicas distintas de las concepciones individualistas de la persona propias del pensamiento occidental (Massó Guijarro 2012; Iniesta Vernet 2009). Se retomará este debate, en intersección con el de género, en la discusión final.

Las cofradías se articulan siempre en torno a la institución denominada dahira. En 1940 se funda la primera y, ya desde sus albores, esta forma de organización hace gala de una formidable capacidad adaptativa. Un buen ejemplo es la dahira Hizbut Tarqiyya, fundada en 1975 en la Universidad de Dakar por estudiantes marxistas (Babou 2002). Algunas de las más representativas hoy son la Matlabul Fawzayni (Guèye 2009;

\footnotetext{
${ }^{12}$ Muridismo como ideología en relación al surgimiento de una intelligentzsia, según este autor.

13 Empleamos en este artículo la concepción del transnacionalismo migratorio según la recoge Suárez Navaz (2008b; ver también Suárez Navaz 2004).

${ }^{14}$ El muridismo ha estado muy vinculado a las elites políticas en Senegal; no en vano los últimos presidentes del país han sido (y lo es el actual) miembros de la cofradía. En cuanto a la ortodoxia oficial de la cofradía respecto de las orientaciones políticas de sus miembros ha oscilado a lo largo de su existencia entre el mayor control (o influencia) y, en los últimos tiempos, una posición de aparente menor control y libertad, enfatizándose la idea de que religión y política son dos asuntos claramente diferenciados. Con todo, el poder generalizado de la cofradía en distintas escalas de la sociedad (redes clientelares con influencia clave en la emisión de visados, posibilitadores de la migración internacional, por ejemplo), es incontestable y reconocido.

15 Sobre la historia y la evolución de la Muridiyya, ver Patterson (1999) o Glover (2007, 2009).
} 
Moreno Maestro 2007) y la Mame Diarra Bousso (la dahira femenina por antonoma$\mathrm{sia}^{16}$ ), ambas fundadas en 1992 y con sedes representativas a escala transnacional.

La dahira constituye, pues, la unidad de organización fundamental en la cofradía murid. Básicamente, es una organización de los fieles en un grupo concreto, con obediencia a un sheikh concreto y una agencia autónoma. En la dahira se cotiza dinero para su redistribución colectiva, se reza, se aprende, se recita, se comparte y se practica cualquier otro asunto de interés colectivo en el contexto que sea. Así, opera tanto como círculo de rezo y socialización como para, en su caso, ejercer presión gubernamental en política, y es tanto centro de solidaridad como sede de poder (Costa Dias 2009: 49). Promueve valores como la disciplina, el trabajo duro, el amor fraternal y el compartir (Babou 2002: 156). Inicialmente nacida como respuesta específica de la migración a ubicaciones urbanas (su proto-forma rural es la daara, una suerte de escuela rural religiosa, colectivista, de trabajo y rezo) (Coumba Diop 1981), la dahira deviene institución educativa e instrumento de socialización e integración, así como una importante fuente de capital social.

Sus funciones en la diáspora migratoria murid se radicalizan en el aspecto de la solidaridad intragrupal y como socialización cosmopolita en la nueva sociedad de acogida. Efectivamente, las dahiras como instituciones con un cierto carácter cosmopolita han sido abordadas por autores como Diouf y Rendall (2000) o Evers Rosander (2000, 2006) para el caso específico de las mujeres. Suelen operar, en este sentido, tanto como vías de intra-integración cuanto de inter-integración (Massó Guijarro 2010, 2011a; Lacomba 2001). Si los vínculos entre los miembros de las dahiras ya son fuertes en Senegal, el sentimiento de marginalidad en la migración los fortifica aún más (Babou 2002: 156; Guèye 2009: 96). Además, en contraste con casos como el soninké o el pulaar, el factor de la etnicidad no juega un rol clave en la organización de vida del murid en la diáspora (Salem 1981). En mi propio trabajo de campo: ¿Tú también eres de Touba? / No, jojalá! Soy de Dakar. Ojalá fuera de Touba". Este tipo de comentarios expresan cómo otro tipo de identidades o lealtades (etnia, parentesco cercano...) se ven relegadas frente a la poderosa significación espiritual de ser murid, encarnada además en la tierra física de Touba como ciudad santa del muridismo.

En este sentido, podemos comprender la dahira como campo social transnacional, a partir del concepto de campo social de Bourdieu: «éste no se limita a un espacio contenedor de redes sociales, sino a un conjunto de dinámicas que emanan del impacto de los procesos de globalización en el mercado laboral y en la gobernabilidad de las poblaciones, cada vez menos arraigadas a un único territorio" (Suárez Navaz 2008b: 785).

Como movimiento sufí que es, el muridismo presenta un aspecto intensamente místico, así como una espiritualidad muy ligada a lo poético y recitativo, al canto y a la música (Skali 2006: 29, 31). Sin embargo, tal dimensión mística de interioridad no está reñida con el apego, como decíamos, al trabajo material y a la laboriosidad, que han de intensificarse en la migración: se emigra para producir riqueza, espiritual pero también material, llegándose a una peculiar confluencia entre utopía y capitalismo (Bava y Bleitrach 1995). Así, la migración en el muridismo se vincula a un matiz de

${ }^{16}$ La mencionada dahira de Mame Diarra constituye, en realidad, una suerte de red de redes de diferentes dahiras repartidas a escala transnacional. 
deber religioso (Ndiggel), de emulación de la migración o hijra del profeta (Glover 2009: 80), lo que se refuerza socialmente con el prestigio alcanzado por la actividad misma de migrar. Hoy incluso en Touba se llega a decir que el talibe que no migre no encontrará esposa ${ }^{17}$.

Las funciones de las dahiras en la diáspora, pues, trascienden con mucho la esfera religiosa, convirtiéndose en auténticas plataformas de integración y participación sociales por cauces distintos de los habituales en las sociedades occidentales, donde la participación política suele entenderse en otras claves. A ello se debe que hablemos de la "ciudadanía a través de la dahira" (ver también Massó Guijarro 2011b), y ya que a través de las dahiras los murid migrados ejercen numerosas funciones y prácticas ciudadanas: trabajo, ocio, redistribución o socialización; distintas maneras de contender y de actuar en la vida pública que, en el caso de la Muridiyya, se realizan a través de las relaciones sociales establecidas en la dahira (Pezeril 2009, Coulon 2009).

\section{MURIDISMO, GÉNERO Y MUJERES}

They don't feel the heat, they don't feel hunger, all they do is praise the Holy Man (Sokhna Fatou Gueye, dicho sobre las mujeres murid) (Roberts, Nooter y Gassia 2003: 159).

El papel de las mujeres en el muridismo ha sido ampliamente abordado desde perspectivas diversas: la agencia real que desempeñan en la práctica, a menudo a través de su protagonismo en los llamados circuitos de intercambio de honor (kersa), vinculados a rituales de ciclo vital (Buggenhagen 2001, 2009) ${ }^{18}$ y de grandísima relevancia en la sociedad murid; los contenidos simbólico-religiosos de las principales figuras femeninas de la cofradía (Coulon y Reveyrand Coulon 1990; Evers Rosander 1997b), con especial énfasis en la única — hasta la fecha- mujer murid que ha ostentado el cargo de califa, Sokhna Magatte Diop (Coulon 1988); la relación de la mujer con los conceptos teológicos de baraka (Coulon 1988) o pureza (Bop 2005); las celebraciones, magal ${ }^{19}$,

\footnotetext{
17 Comunicaciones personales de los interlocutores.

18 Ver Talpade Mohanty (2008a: 134) para un análisis interesante, desde un feminismo poscolonial, de los intercambios asociados a rituales del ciclo vital. Buggenhagen (2009) reclama que los estudios hechos por varones normalmente han restado importancia a estos fenómenos, cuando ameritan una crucial relevancia en la sociedad wolof y murid; en sus propios términos, tales académicos han infraanalizado las palabras de las mujeres, con lo cual automáticamente han infraanalizado las formas de riqueza que ellas manejan (Buggenhagen 2009: 201). La autora considera este error de índole epistemológica; en los términos de Coulon (1988: 115), la "incapacidad" para percibir o aprehender estas cuestiones. Buggenhagen (2009) también denuncia esta devaluación de los rituales asociados con la reproducción en los análisis occidentales, recogiendo la crítica ejercida a su vez por Annette Weiner. Considera que restarles importancia escamotea la verdadera realidad de la relevancia que estas prácticas rituales poseen en sus sociedades de origen, en tanto que se consideran las vías de conexión de lo individual con lo divino.

${ }^{19}$ Los magal son las grandes celebraciones religiosas murid por antonomasia, que suelen involucrar una comida o cena colectiva y, sobre todo, el honramiento a un líder religioso concreto. Estos eventos (lugares-momentos, los llama Guèye) son esenciales para la construcción del carisma de tales líderes (Guèye 2009: 105). Suelen estar ligados a fechas clave en el calendario musulmán, pero incluso estas fechas resultan a menudo modificadas y matizadas por las propias efemérides murid.
} 
peregrinaciones y dahiras esencialmente femeninas (Coulon 1999) ${ }^{20}$; y, finalmente, la existencia de algunas marabouts mujeres ${ }^{21}$ (Gemmeke 2009).

La figura en torno a la cual se arracima el culto femenino en el muridismo es Mame Diarra Bousso (o Shokha Diarra), la madre de Ahmadou Bamba, fundador de la orden. El énfasis otorgado a la importancia de Mame Diarra es crucial: se le atribuye de forma genuina la responsabilidad, el mérito de que exista el muridismo, tanto en un sentido biológico (ella parió a Ahmadou Bamba), como en un sentido moral (ella lo educó, lo socializó, constituyéndolo como él fue). Es un hecho constatado la responsabilidad atribuida a las mujeres en el éxito o el fracaso de su prole, frente a la absoluta irrelevancia del papel del padre con respecto a ello. La reproducción, considerada tarea por antonomasia de las mujeres, no se comprende solamente en un sentido biológico, sino que se enfatiza el aspecto de la socialización, la endoculturación en el muridismo y su transmisión (Roberts, Nooter y Armenian 2003b: 151). Ello se vería, desde una óptica occidental, como una subordinación femenina ya que se "obliga" a las mujeres a determinada tarea; otra lectura bien distinta sería entender que existe una parcela de poder exclusiva de las mujeres, donde los hombres carecen del mismo. La responsabilidad es siempre, también, poder. Así, la cuestión del poder genealógico resulta crucial para elaborar el discurso murid sobre el poder y la valía de las mujeres, y enlaza con el culto femenino a la maternidad preislámico en Senegal y en muchos lugares de África.

El culto a la maternidad se expresa así a través de la veneración a la figura de Mame Diarra, llegándose a la afirmación de que "Ils son trois, rien que trois: Dieu, son prophète Mohamed et Mam Diarra" (en Evers Rosander 2003a: 297). Las seguidoras murid son fieles devotas de Mame Diarra y profundas conocedoras de todos los milagros ${ }^{22}$ a ella atribuidos por tradición oral y de los numerosos ejemplos de virtud recontados en su breve vida (falleció a los 33 años). No es baladí considerar la presencia y la significación de figuras femeninas, como la María cristiana (Maryam en el Corán) en textos clásicos sufíes como los de Rumi, quien afirma que el alma del místico es semejante a María: "Si tu alma es lo bastante pura y está lo bastante llena de amor, se vuelve como María: engendra al Mesías" (en Skali 2006: 89). Aquí encontramos un paralelismo evidente de las figuras de María y Jesús con las de Mame Diarra y Ahmadou Bamba: su alma, pura y llena de amor, engendró al fundador del muridismo. En el propio Corán, "Nuestro cuerpo es como María: cada uno de nosotros lleva a un Jesús en su interior, pero mientras los dolores del alumbramiento no se manifiestan en nosotros, nuestro Jesús no nace" (Corán 19, 22-26, en Skali 2006: 89).

Así, tal relevancia concedida a las mujeres en el sufismo clásico contempla su contraparte en el sufismo específico de la Muridiyya precisamente en el culto a Mame Diarra, y desafía nuevamente las asunciones sobre la subordinación de la mujer en

\footnotetext{
${ }^{20}$ Si bien el estudio de Mahmood (2008) está referido a Egipto, resulta muy interesante como contraste considerar sus conclusiones sobre el movimiento de mujeres islámicas, que abordó como agente activo y respuesta femenina a la praxis hegemónica.

${ }^{21}$ Se ha de matizar que las marabouts mujeres estudiadas por Gemmeke pertenecían a la cofradía Layenne. Sin embargo, en mi trabajo de campo hasta la fecha he hallado la afirmación frecuente de que "hay muchas mujeres marabouts".

${ }^{22}$ Para comprender la relevancia de los milagros como soportes de enseñanza en el sufismo, ver Skali (2006: 83ss).
} 
el islam. De Mame Diarra la voz popular proclama los valores considerados más elevados en el muridismo (y en el sufismo y el islam), como estar investida de baraka (una de las críticas habituales a la subordinación de las mujeres en el islam es que éstas no puedan participar de la baraka), las extremas competencia y precocidad (a los diez años) en la lectura del Corán, la hospitalidad ilimitada, la capacidad de estar toda una noche rezando, la legendaria discreción, la paciencia o el autosacrificio ("sutura" en wolof). Mame Diarra es venerada como una "santa por derecho propio" (Roberts, Nooter y Armenian 2003b: 155) y su mismo nombre, "Diarra", es considerado una bendición, ya que significa "quien vive con Dios" (Roberts, Nooter y Armenian 2003b: 157).

Tales excepcionalidad y santidad como mujer poseen, además, una potente raigambre genealógica: le vienen de antiguo, por así decir, en tanto que también se afirma que ya su madre (es decir, la abuela del fundador del muridismo) fue una mujer absolutamente atípica (Roberts, Nooter y Armenian 2003: 155): aprendió el Corán a una edad muy temprana y lo transmitió con éxito a muchas personas (de nuevo, habilidades de gran predicamento entre los fieles murid); vivió ciento treinta años; vestía "como un hombre" y mostraba la inigualable capacidad (solo reservada a los más especiales, a los más "amigos de Dios") de poder estar "una noche entera rezando sin mostrar cansancio o necesidades de ningún tipo". Por tamañas bondades fue bendecida con Mame Diarra... y ella a su vez lo fue con Ahmadou Bamba.

Curiosamente, esta suerte de transmisión genealógica de valores y santidad se extiende a los espacios territoriales, físicos: si el lugar que vio nacer a Mame Diarra es el pueblo de Porokhane, y Mame Diarra engendró a Ahmadou Bamba, entonces Porokhane engendró (y es su causa genética, su etiología) a Touba, la ciudad santa del muridismo. "Mais c'est Porokhane qui a engendré Touba, donc c'est Porokhane qui est tout, quie es la vie [...] Que dire donc de Porokhane qui est mére de Touba" (Evers Rosander 2003a: 315). Porokhane y Mame Diarra se simbolizan mutuamente, y ella es la madre de la religión, no solamente en un sentido biológico. Evers Rosander (2003a: 316) considera, pues, que tierra y cuerpo se funden en el mausoleo de Mame Diarra en Porokhane, y que ello posee un alcance cosmológico, espiritual y metafísico: Prokhane deviene geografía sagrada; las mujeres han creado su propia cosmología sufí atravesada por el espacio y el cuerpo sagrados.

Me parecen fundamentales en este imaginario popular tan potente los elementos maternales y genealógicos. Es importante reparar en que no son el padre ni el abuelo de Ahmadou Bamba los que se glosan, los que se consideran portadores de tanta gracia, transmisores de tamaña baraka. Resulta crucial que sea la rama femenina la que se enfatiza en el muridismo como creadora, artífice y transmisora del valor divino que ha dado lugar a la cofradía. De hecho, desde su análisis feminista, Evers Rosander reseña que muchas mujeres consideran que hay una ruptura de la dominación masculina y una ganancia de estatus social para la mujer a través de la figura de Mame Diarra, que ha "elevado su rango": "Quand nous allons à Porokhane avec notre association Mam Diarra, cést nous les femmes, qui sommes confortablemente installées à lávant du car, et les hommes son à l'arrière [...]" (Evers Rosander 2003a: 309).

Las dahiras femeninas (las denominadas "Dahiras de Mame Diarra"), exclusivamente compuestas, lideradas y financiadas por mujeres, son habituales y numerosísimas tanto en Senegal como en la diáspora migratoria murid, y muestran un infatigable e incisi- 
vo dinamismo. Como se ha reseñado, las mujeres murid ya acreditaban una larga costumbre de asociacionismo a nivel tradicional en el espacio rural, pero en la traslación al ámbito urbano durante las migraciones de los años 50 en Senegal —igual que en el caso de los hombres- es cuando sus nivel de actividad en las dahiras se intensifica (Piga 2003). Su modo de organización y funcionamiento es similar al de las dahiras masculinas; presentan sin embargo especificidades, en el culto femenino en su base y en el tipo de asuntos de los que se ocupan. Allí se hallan sus rasgos identitarios.

Los asuntos a los que suelen dedicar más énfasis las dahiras femeninas son precisamente los relacionados con esferas re-productivas y de cuidado, tanto a escala micro como a escala transnacional (por ejemplo, la financiación de proyectos de casas de acogida a menores de familias sin recursos), transcendiendo pues, con mucho, la esfera de la familia nuclear; e igualmente vinculados, siempre de modo íntimo, con la figura de Mame Diarra, su mausoleo en el pueblo de Porokhane en Senegal (que constituye un peregrinaje propio con una dimensión política, Evers Rosander 2003a: 301; 2003b) y sus festividades propias, como el Magal de Porokhane (Guèye 2009), también llamado "Magal de las Mujeres". Podemos afirmar que las mujeres murid en la diáspora ${ }^{23}$, a través del culto a Mame Diarra y sus eficaces cotizaciones económicas, gestionan una interesante forma de cuidado transnacional, o cadena transnacional del cuidado, ya que de hecho financian proyectos destinados al sustento de personas más allá de su esfera familiar directa y extensa (al respecto son de candente actualidad en el contexto nacional español los estudios de Vázquez Silva 2010; para otros contextos transnacionales, ver también $\mathrm{Ba} 2008^{24}$ ).

Finalmente, y realizando un ejercicio sintético, encuentro que las interpretaciones académicas sobre el papel de las mujeres murid en la cofradía oscilan entre dos grandes tendencias hermenéuticas ${ }^{25}$, a saber: las que consideran que existe un margen amplio para la agencia femenina y que, de hecho, existen numerosos ejemplos del activo papel de las mujeres murid en la cofradía, a través de estrategias que limitan el poder patriarcal del liderazgo masculino; y las que consideran que, pese a los esfuerzos interpretativos anteriores, el papel de la mujer continúa siendo de una gran subordinación en el muridismo, primando la marginación y la desigualdad (a favor del hombre) en la cofradía.

\footnotetext{
${ }^{23}$ Sobre actividades (re)productivas de mujeres murid en la diáspora, ver Babou (2009) o Evers Rosander (2006).

${ }^{24}$ Los trabajos de $\mathrm{Ba}$ (2008) analizan la renegociación de la identidad musulmana en la migración (en su caso, para el contexto de Nueva York). No es el objetivo de este artículo analizar el elemento de tal renegociación en el caso de las mujeres murid en Madrid; elemento que, sin embargo, aparece como es inevitable y propio de cualquier agencia en contexto migratorio.

${ }^{25}$ No se vuelven a citar en este apartado los nombres de tales autores en aras del afán sintético de este párrafo; sin embargo, nos referimos a los enfoques que se han ido citando y desagregando en los párrafos anteriores.
} 


\section{A MODO DE EJEMPLO CONTEXTUAL: LA DAHIRA DE MAME DIARRA EN MA- DRID $^{26}$}

Nos gustó mucho tener el reconocimiento, el respeto de los chicos por el trabajo tan grande que hicimos con ese primer magal (interlocutora murid).

La dahira femenina en Madrid nace en 2008, como respuesta a la inquietud mostrada por Mame Mor, uno de los sheikhs más relevantes que realizan viajes recurrentes en la diáspora (fallecido el 29 de junio de 2012), en una de tales visitas regulares a sus fieles. De hecho, es frecuente que los sheiks murid animen a las mujeres a fundar sus propias dahiras, y ya Coulon (1988) reseñó la fuerza emancipadora subyacente a este hecho. Mame Mor declaró públicamente que la activa presencia y la intensa actividad de las mujeres en los magal y en la organización de la cofradía en Madrid merecía, por derecho, la constitución de una esfera autónoma encarnada en la dahira; en cierto modo, para que su labor no quedara invisibilizada en el conjunto de la dahira de los hombres. Insta pues al colectivo femenino a la fundación de una dahira independiente de los hombres como vía de adquisición de autonomía y de emancipación práctica. Y, sin duda, la fundación de la dahira en Madrid ha constituido, en voz de sus propias protagonistas, una importante forma de empoderamiento.

En cuanto al culto a Mame Diarra, el específico de todas las dahiras femeninas murid, las mujeres en Madrid profesan efectivamente la intensa fidelidad a esta figura de virtud y perfección, enumerando con frecuencia los mitos populares sobre sus inmensos valores y las historias que tradicionalmente se cuentan sobre su vida, de la que son grandes conocedoras.

Así pues, en 2008 comienza la organización para conformarse como dahira. Al carecer de experiencia previa en la diáspora, las fundadoras atravesaron unos primeros momentos de búsqueda de asesoramientos diversos y organización logística. Se escogen las figuras oficiales de la presidenta, la vicepresidenta, la secretaria y la tesorera. Intentan registrarse como asociación (a través de la amiga de una de ellas, que trabajaba en una gestoría) pero, ante la complicación burocrática que no se sienten capaces de afrontar en aquel momento, aplazan el trámite, que continúa hoy en suspenso.

Comienzan la cotización regular y las reuniones bimensuales (dos domingos de cada mes) en la Casa de Serigne Touba $^{27}$ a las afueras de Madrid, destinadas como es habitual a funciones varias: el encuentro como vía de socialización y compartir experiencias, sentimientos, etc.; el estudio de los poemas de Serigne Touba y de los versos coránicos; el canto y recitado de poemas (el kourel ${ }^{28}$ ) como vía, tanto para la

${ }^{26}$ La información recogida en este epígrafe procede directamente de dos fuentes fundamentales: mi propia observación participante en la comunidad murid desde enero de 2011 y la entrevista semiestructurada en profundidad con AK, tesorera de la dahira desde 2008, en Madrid (25/ 01/2012).

${ }^{27}$ La denominada Casa de Serigne Touba es un chalet en una urbanización a las afueras de Madrid (se omite más detalles sobre el emplazamiento exacto por respeto a sus usuarios) que la propia comunidad murid construyó y financió para contar con un espacio adecuado a sus necesidades durante los magal, las grandes celebraciones rituales, la acogida de los marabouts durante sus visitas, etc. Funciona con regularidad desde 2008.

${ }^{28}$ Círculos místicos de rezo y canto. 
expansión y conocimiento mutuo, como para el acercamiento a la divinidad, según el camino esotérico del sufismo ${ }^{29}$. Además, se enorgullecen de que sus kourel son tan emocionantes como los de los hombres.

La ocupación por parte de las mujeres de la Casa de Serigne Touba ya posee un elemento de empoderamiento importante. Inicialmente este lugar de reunión era gestionado exclusivamente por hombres: la organización de los tiempos, la distribución o el uso del lugar, todo ello era asunto y decisión masculina. Las mujeres recibían, cuando eran precisos su concurso o su ayuda, noticias y peticiones sin mayor poder consultivo, por así decir ("en cualquier momento te llamaban y te decían 'tal día a tal hora os necesitamos...', como si no tuviéramos otra cosa que hacer que estar sentadas a esperar su llamada... ahora esto ya no es asín). En cambio, al comenzar la dahira de las mujeres su andadura cambia la situación a un mayor control por su parte: ellas también expresan su calendario festivo, por así decir, y sus necesidades en el uso y la gestión de espacios. La casa mejora enormemente - cuentan- en cuanto a pulcritud y orden, estableciéndose turnos y la norma de una limpieza general tras los magal. Especialmente, obtienen el reconocimiento de los hombres cuando celebran, tras ímprobos esfuerzos organizativos, el primer magal de Porokhane de forma independiente, logrando una elevada recaudación para enviar a diversos proyectos a Senegal. Según sus propias voces, fue muy grato obtener el "reconocimiento de los chicos". Expresan haber tenido mayores dificultades en la organización del segundo magal, al año siguiente, pero que incluso entonces lograron, de nuevo tras una titánica cantidad de trabajo e implicación, celebrarlo y recaudar una cantidad significativa para su redistribución en los diversos proyectos en los que colaboran.

Es importante considerar que, previamente a la constitución de la dahira femenina independiente, las mujeres formaban parte de la dahira de los hombres, que podría definirse casi como mixta por defecto, en el sentido de que, según cuentan las mujeres y como se mencionó más arriba, su poder decisorio y de gestión era inexistente: se contaba con su fuerza de trabajo y su concurso, pero sin previa consulta, sino dándose por hecho que debían obedecer en el acto sin más. En cierto modo, el papel y la presencia de estas mujeres quedaban subsumidas o difuminadas en la presencia masculina y su dominancia por defecto y costumbre ${ }^{30}$. La escisión de una dahira de mujeres, instada además por un marabout hombre, supone pues una clara constitución de autonomía, la emergencia de un campo autónomo para la acción.

Desde 2008 la dahira ha evolucionado en varios sentidos. Al comienzo estaba formada por más mujeres, muchas de ellas recién llegadas de Senegal que buscaban a

\footnotetext{
${ }^{29}$ En este camino esotérico los aspectos emocionales resultan clave para el alcance de determinados estados espirituales. Las sesiones de invocación colectiva ( $d h i k r$ ) se orientan a esta exaltación del sentimiento de la trascendencia, a menudo vinculada al lugar simbólico del corazón como centro sutil del ser (Skali 2006: 34-36). En mi trabajo de campo he observado la frecuencia con que los murid describen sus sentimientos religiosos tocándose el corazón y apelando a que "hay que sentirlo, lo sientes aquí, y la imposibilidad de ofrecer una respuesta o explicación racionales a ciertas creencias.

${ }^{30}$ Ello no parece exclusivo del caso concreto que se expone aquí: "Por ejemplo, en mi campo me sale que en las dahiras mixtas las mujeres estaban bastante molestas por lo limitado de su papel [...]", cuenta Iria Vázquez Silva (comunicación personal, 16/02/2012) de su propia experiencia en el campo.
} 
través de esta vía una forma de socialización. Igualmente se contaba con más presencia de mujeres de edad madura (entre cuarenta y cincuenta), mientras que en la actualidad se ha incrementado la participación de jóvenes, entre los veinte y los treinta y cinco, con y sin hijos, solteras y casadas, trabajando fuera y dentro del hogar. En estos aspectos, pues, el perfil de las personas miembros ha sido plural desde el inicio.

La crisis económica actual también ha afectado a la participación de las mujeres en la dahira, si bien ni con respecto al presente ni con respecto al inicio pueden estimar un número concreto y cerrado de miembros de la dahira, ya que fluctúa en gran medida y, además, hay que diferenciar entre las personas que solo cotizan y las directamente activas en el movimiento. En el aspecto de las cotizaciones, parece que hay problemas recurrentes para su recaudación, en tanto que muchas mujeres (y sus maridos, de haberlos) tienen ahora más problemas para encontrar trabajos, sumando a ello el hecho de que muchas no tienen papeles, hallándose en situaciones de irregularidad jurídica que precariza los empleos a los que puedan tener acceso.

Si seguimos la desagregación que Dekker y Uslaner (2001:12) han realizado del capital social (como bien público o cooperación) en cuatro funciones distintas, constatamos que la dahira femenina en Madrid las desempeña sin ambigüedad: compartir información, coordinación de actividades, formación de decisiones y ejecución de tales actividades a niveles macro o transnacional. Además, concibiendo el capital social principalmente como las relaciones, o el "conjunto de redes sociales que un actor puede movilizar en provecho propio" (Rist 2000: 139), resulta evidente que las mujeres a través de la dahira son grandes movilizadoras de relaciones y, por tanto, de capital social. Por otro lado, es obvio que éste, una herramienta intangible por así decir, genera recursos y riqueza, tanto inmateriales como materiales. Así, vemos cómo en la dahira las mujeres recaudan y gestionan bienes monetarios de modo autónomo; financian proyectos de cooperación (y cuidado transnacional) en Senegal; gestionan el uso del espacio (simbólica y prácticamente crucial) de la Casa de Serigne Touba según sus necesidades; y organizan su propia agenda de servicio comunitario (a través de celebraciones), que ellas definen como clave para mostrar al colectivo transnacional murid que son fieles consecuentes con sus creencias y que están trabajando en "este mundo" en aras de su futuro en "el siguiente". Ello implica el poder de manejar el propio destino espiritual, cuestión que no es precisamente baladí. Así, no se trata solo de servir a la mayor gloria de Ahmadou Bamba, sino, muy especialmente, escoger cómo se sirve, cuándo y de qué manera, sin recibir órdenes salvo del propio y elegido marabout (según la clásica obediencia sufí).

\section{DISCUSIÓN Y CONCLUSIONES: ¿SALVAR A LAS MUJERES DE SÍ MISMAS?}

They are active Muslims, even if their practices are informal, hidden, parallel, or heterodox; hence it is wrong to relegate the female Muslim universe to this twilight zone where it only appears to belong because of our inability to study it (Coulon 1988: 115).

Are woman aware of their marginalization? (Bop 2005: 1102).

Como se indicó más arriba, existen dos grandes tendencias en los análisis sobre muridismo, a saber: la interpretación (oyendo su propia voz) del muridismo desde 
su perspectiva y su lógica internas (llamémoslo la "visión emic") y la interpretación presuntamente neutral y estructural (la "visión etic ${ }^{31}$ "). Anticipo que ésta última se me antoja mucho más pertrechada de ideología occidental, individualista y neoliberal que de neutralidad. Tales dos visiones se aplican a las dos grandes "contiendas por la igualdad", o esferas susceptibles de discutirse en la dialéctica dominación-emancipación, presentes en el muridismo: la relación marabout-talibe y la relación hombre-mujer (o bien liderazgo murid casi siempre masculino-mujeres, como colectivo). En este trabajo me he centrado en la segunda esfera de contienda, por así decir, que motivaba de hecho la reflexión, aunque ambas esferas se hallen íntimamente relacionadas y necesiten por ello una discusión enlazada.

La pregunta inicial era si fenómenos como la dahira de Mame Diarra en Madrid desafíaban el patriarcado murid. Soy consciente de que esta inquietud, el título mismo escogido para este trabajo, se hacen eco de uno de esos "debates blancos", blancos por su origen y por su ingenuidad. ¿Desafio al patriarcado murid? Tal pregunta también es blanca, si asumimos las reflexiones de Oyewumi (2002) sobre la base conceptual de las críticas del feminismo blanco, y habría de ser probablemente reformulada en unos términos más socialmente informados. En todo caso, resulta evidente que aquella desafiante pregunta inicial ha operado más como escoplo para la discusión que como acicate para una contestación definitiva. Aquí se ha tratado, precisamente, de ofrecer una problematización de tal pregunta desde la asunción de su complejidad. Por otra parte, es evidente que no podemos reducir el debate a una cuestión de color. Hablamos de feminismos "negros" y "blancos" como conceptualizaciones hermenéuticas, pero no podemos ignorar hechos como que sea precisamente Bop (2005), una senegalesa, la que denuncia precisamente la relegación de la mujer en el muridismo y minimiza la importancia de las esferas de agencia autónomas de las mujeres en la cofradía.

Asumo la diferenciación entre las llamadas dos grandes "arenas societales" (Anthias 2012): la organizacional (de la acción y la intersubjetividad) y la discursiva (de la experiencia y la auto-narración). Asumo que ambas constituyen dos niveles diferentes de análisis que ameritan el particular interés de la persona investigadora. Sin embargo, en este trabajo me he inclinado, como antropóloga, por el énfasis del discurso, de la narración ("emic"), y dado que ya abundan los estudios, citados aquí, que pretenden una interpretación más acertada de la que las propias interlocutoras ofrecen de sí mismas y sus propias vidas.

Por otro lado, siguiendo a Estrada Díaz ${ }^{32}$, "la espiritualidad, el fraude del poder dominante y la elección libre no se oponen sino que pueden formar una mezcla".

${ }^{31}$ Se ha de realizar una aclaración importante en torno al uso de la dicotomía "emic/etic" en estos momentos. Las visiones emic/etic, clásicas en antropología, se consideran necesarias en su conjunción para la formulación de conclusiones científicas. Aquí realizamos un uso laxo de tales términos, considerándolos y adaptándolos a este contexto en un sentido lato: lo "emic" es, básicamente, la interpretación de los propios actores y en su propia voz; lo "etic" es el análisis según conceptos propios que han realizado ciertos autores sobre muridismo, que, en cierto modo, corrigen las hermenéuticas autóctonas. Así, el empleo en este artículo y en este momento de la dicotomía "etic/emic" no se hace eco de la ortodoxia disciplinaria al respecto en antropología, y obedece fundamentalmente a la matización recién realizada.

32 Comunicación personal, 17/02/2012. 
Ello supone asumir que tal vez la respuesta compleja a aquella pregunta compleja no sea una afirmación o una negación absolutas, sino más bien un reconocimiento de la pluralidad y de la heterogeneidad existente en las relaciones de poder, tanto entre mujeres y hombres murid, como entre discípulos y marabouts murid. Si pensamos, por ejemplo, en la relación entre un discípulo masculino y la sheikh femenina del muridismo, ¿cuál es la encrucijada de poder en que nos hallamos? ¿Qué sumisión encontramos ahí, más o menos conveniente, más o menos aceptable según nuestra óptica? ¿Entendemos que la "sumisión" —acaso no sea tal— de este hombre es más aceptable que la de una mujer en situaciones análogas? ¿Por qué? ¿Acaso no estamos, de este modo, concediendo más crédito a la capacidad de escoger de un hombre que a la de una mujer, si damos por hecho que en un caso hablamos de libre opción y en el otro de ciega sumisión? Pienso, pues, que las diversas opciones e interpretaciones aquí manejadas no son excluyentes. Así, Buggenhagen (2009: 192) considera que las mujeres murid son personas racionales que "invierten" espiritualmente en su beneficio. Efectivamente, ello no es incompatible con las creencias tanto materiales como inmateriales de estas murid. Bien al contrario, el muridismo muestra una interesante confluencia entre los intereses "del cielo" y "de la tierra" por así decir, que transita desde una intensa dedicación al comercio y al atesoramiento de bienes materiales hasta una espiritualidad mística.

Para muchas mujeres el hecho de que las mujeres murid cocinen durante horas y días en los magal constituye un sometimiento, por ejemplo. Para las protagonistas del hecho, sin embargo, constituye una vía privilegiada de servicio a la divinidad y por ende, también, de socialización y ganancia de bienestar global. Añadimos además que algunos hombres también cocinan. ¿Cuál es la interpretación correcta? Esta pregunta me la hago como filósofa, con una conciencia normativa ética de las cuestiones. Sin embargo, la respuesta inevitablemente la pienso como antropóloga: la interpretación válida es la emic, la propia o autóctona ${ }^{33}$ o de "VOz en primera persona", si queremos evitar la ventriloquia social ${ }^{34}$, en el mejor de los casos, o el imperialismo epistemológico occidental, en el peor. No querría caer en la "actitud paternalista y condescendiente" que Bell Hooks (Jabardo Velasco 2008: 42) encontraba ya en las feministas blancas. Y, con todo, bien vale recordar a Talpade Mohanty (2008a: 147) cuando precisa que "A menudo la mera existencia de una división sexual del trabajo se toma como prueba de la opresión de las mujeres en varias sociedades. Ello procede de la confusión y mezcla de los potenciales descriptivos y explicativos del concepto de división sexual del trabajo". Efectivamente, pienso que en numerosos análisis feministas-blancos hallamos una descripción no problematizada de los trabajos o tareas "que importan" (los socialmente prestigiados) y los que no, sin cuestionamiento alguno de por qué algo importa y no importa, de quién y por qué hace lo que importa y lo que no importa. Muchas veces, incluso hallamos la automática consideración (insisto, sin problematización epistémica alguna) de que una tarea concreta no es socialmente prestigiosa cuando en realidad sí lo es en la sociedad analizada, y tal juicio de desprestigio se debe a una presbicia cultural de la investigadora (como refleja la cita de Coulon que encabeza estas conclusiones).

\footnotetext{
${ }^{33}$ Según, insisto, la matización realizada previamente sobre el uso de la dicotomía "emic/etic".

${ }^{34}$ Según el uso del término en Bretón Solo de Zaldívar (2008).
} 
Las dos grandes tendencias hermenéuticas sobre el papel de la mujer en el muridismo, arriba mencionadas, son analógicas pues a las que existen en las interpretaciones sobre la cofradía misma en su estructura religiosa y de poder: el muridismo desde su propia perspectiva como vía de acercamiento a la divinidad y lo absoluto, o bien el muridismo como un "fraude" para explotar de modo capitalista a los discípulos $^{35}$. Podemos decir, siguiendo el enfoque de la interseccionalidad (Anthias 2012 ${ }^{36}$ ), que en estas dos tendencias enfrentadas se entrecruzan los clásicos ejes del género y la religión, que operan, pues, tanto a nivel de las relaciones de género (hombre/mujer) como en las relaciones religiosas (marabout/talibe). En mi opinión podemos verlas en clave antropológica como el "emic" y el "etic" de la cuestión: el "emic" de sus propias voces o el "etic" de la voz de la investigadora. Con todo, estimo que las tendencias descritas continúan hallándose hoy en una tensión irresoluta; sin embargo, aunque constatemos que algunos registros aparentan inconmensurables, el hecho de hacerlos conscientes ya es subversivo o transformador en sí mismo.

\section{BIBLIOGRAFÍA CITADA}

Anthias, F. 2012. "Beyond social categories: different levels of analysis and intersectional framings". Conferencia en el I Congreso Internacional FEMIGRA: Feminismo, migración e intervención social. Barcelona, 9-11 febrero 2012.

Ba, A. 2008. "Les femmes mourides à New York - Une renégociation de l'identité musulmane en migration", en Diop, M. C. (ed.), Le Sénégal des migrations: 389-408. París: Khartala.

Babou, Ch. A. 2002. "Brotherhood solidarity, education and migration: the role of the Dahiras among the Murid Muslim community in New York". African Affairs 101: 151-170.

Babou, Ch. A. 2003. "Educating the Murid: Theory and Practices of Education in Amadu Bamba's Thought. Journal of Religion in Africa 33-3: 310-327.

Babou, Ch. A. 2009. "Money, "Caste" Gender, and Social Status among Senegalese Female Hair Braiders in the United States". Africa Today 55-2: 3-22.

Bava, S. y Bleitrach, D. 1995. "Les murid entre utopie et capitalisme". Le Mond Diplomatique, noviembre 1995: 21.

Beck, L. J. 2001. "Reining the marabouts? Democratization and local governance in Senegal". African Affairs 100: 601-621.

Bop, C. 2005. "Roles and the position of women in Sufi brotherhoods in Senegal". Journal of the American Academy of Religion 73-4: 1099-1119.

Brenner, L. 2005. West African Sufi. The religious heritage and spiritual search of Cerno Bokar Saalif Taal. Londres: Hurst \& Co.

Bretón Solo de Zaldívar, V. 2008. "De la ventriloquia a la etnofagia o la etnitización del desarrollo rural en los Andes ecuatorianos", en Martínez Mauri, M. y Rodríguez Blanco, E. (eds.), Intelectuales, mediadores y antropólogos: la traducción y reinterpretación de lo global en lo local: 113136. Donostia: Ankulegi.

${ }^{35}$ Se ha criticado en estos análisis economicistas y materialistas que no conceden el peso suficiente a la religiosidad de sus interlocutores, concentrándose más en la estructura que en el significado (Buggenhagen 2009: 192; Iniesta Vernet 2009).

${ }^{36} \mathrm{El}$ enfoque de la interseccionalidad constituye una herramienta heurística clave para estas reflexiones, en tanto que se incrusta en un feminismo antirracista y comprometido con la lucha contra la subordinación, pero asumiendo, asimismo, un rechazo al feminismo blanco de hechura epistemológica monolítica y anclado en la concepción exclusivamente occidental del mundo. En los términos de Erica Burman (2012), la interseccionalidad nos permite (y nos obliga a) interrogar analíticamente las categorias. 
Buggenhagen, B. A. 2001. "Prophets and profits: gendered and generational visions of wealth and value in Senegalese Murid households". Journal of Religion in Africa 31-4: 373-401.

Buggenhagen, B. A. 2009. "Beyond brotherhood. Gender, religious authority, and the global circuits of Senegalese Muridiyya", en Diouf, M. y Leichtman, M. A. (eds.), New perspectives on Islam in Senegal. Conversión, migration, wealth power, and femininity: 189-210. Nueva York: Palgrave McMillan.

Burman, E. 2012. "Feminist perspectives on researching violence against women and children". Conferencia en el I Congreso Internacional FEMIGRA: Feminismo, migración e intervención social. Barcelona, 9-11 febrero 2012.

Copans, J. 1988. Les marabouts de l'arachide. La confrérie mouride et les paysans du Sénégal. París: L'Harmattan.

Copans, J. 2000. «Mourides des champs, mourides des villes, mourides du téléphone portable et de l'internet". Afrique Contemporaine 194: 24-33.

Costa Dias, E. 2009. "Cofradías musulmanas y movimientos de Da'wa: dos concepciones del Islam en África occidental", en Iniesta Vernet, F. (ed.), El Islam del África negra: 39-60. Barcelona: Bellaterra.

Coulon, Ch. 1981. Le Marabout et le Prince: Islam et pouvoir au Senegal. París: Pedone.

Coulon, Ch. 1988. "Women, Islam, and Baraka, en Cruise O'Brien, D. y Coulon, Ch. (eds.), Charisma and brotherhood in African Islam: 113-134. Oxford: Clarendom Press.

Coulon, Ch. 1999. "The Grand Magal in Touba: a religious festival of the mouride brotherhood of Senegal.. African Affairs 98: 195-210.

Coulon, Ch. 2009. "Las dinámicas del islam en el África negra en el umbral del siglo XXI: entre lo local y lo global, el islam como 'derivación', en Iniesta Vernet, F. (ed.), El Islam del África negra: 61-80. Barcelona: Bellaterra.

Coulon, Ch. y Reveyrand Coulon, O. 1990. L'Islam au féminin: Sokhna Magat Diop, cheikh de la confrérie mouride (Sénégal). Burdeos : Institut d'Études Politiques.

Coumba Diop, M. 1981. "Functions and Activities of the Murids' Urban 'Dahira' in Senegal». Cahiers d'Études Africaines 21-81/83: 79-91.

Creevey, L. E. 1991. "The impact of Islam on women in Senegal". The Journal of Developing Areas 25-3: 347-368.

Crespo Ubero, R. 2006. "Participación y asociacionismo senegalés. De la visibilidad a la conexión transcontinental", en Jabardo Velasco, M. (ed.), Senegaleses en España. Conexiones entre origen $y$ destino: 132-142. Madrid: Ministerio de Trabajo y Asuntos Sociales.

Cruise O’Brien, D. B. 1969. "Le talibé mouride: Étude d'un cas de dépendance sociale». Cahiers d'Études Africaines 9- 35: 502-507.

Cruise O'Brien, D. B. 1970. "Le talibé mouride: La soumission dans une confrérie religieuse sénégalaise». Cabiers d'Études Africaines 10-40: 562-578.

Cruise O'Brien, D. B. 1988. "Charisma comes to town: Mouride urbanization 1945-1986", en Cruise O'Brien, D. B. y Coulon, Ch. (eds.), Charisma and brotherhood in African Islam: 135-156. Oxford: Clarendom Press.

Cruise O'Brien, D. B. 2003. Symbolic confrontations. Muslims imagining the State in Africa. Londres: Hurst \& Co.

Dekker, P. y Uslaner, E. M. 2001. Social capital and participation in everyday life. Londres: Routledge.

Diouf, M. y Leichtman, M. A. 2009. "Introduction. New perspectives on Islam in Senegal. Conversión, migration, wealth power and femininity", en Diouf, M. y Leichtman, M. A. (eds.), New perspectives on Islam in Senegal. Conversion, migration, wealth power, and femininity: 1-20. Nueva York: Palgrave McMillan.

Diouf, M. y Rendall, S. 2000. "The Senegalese Murid trade diaspora and the making of a vernacular cosmopolitanism". Public Culture 12-3: 679-702.

Evers Rosander, E. 1996. "Workshop on Women and Sufism in Africa". Uppsala, 2-4 septiembre 1996.

Evers Rosander, E. 1997a. "Introduction: The Islamization of 'Tradition' and 'Modernity', en Evers Rosander, E. y Westerlund, D. (eds.), African Islam and Islam in Africa: 1-27. Londres: Hurst \& Company. 
Evers Rosander, E. 1997b. "Le dahira de Mam Diarra Bousso à Mbacké: analyse d'une association religieuse de femmes sénégalaises", en Evers Rosander, E. (ed.), Transforming female identities: women's organizational forms in West Africa: 160-174. Uppsala: Nordiska Afrikainstitutet.

Evers Rosander, E. 2000. "Money, marriage and religion: Senegalese women traders in Tenerife, Spain", en Salter, T. y King, K. (eds.), Africa, Islam and development: Islam and development in AfricaAfrican Islam, African development: 167-191. Edimburgo: University of de Edinburgh.

Evers Rosander, E. 2003a. "Mam Diarra Bousso la bonne mère de Porokhane, Sénégal". Revista Trimestrale di Studi e Documentazione dell'Istituto Italiano per l'Africa e l'Oriente LVIII 3-4: 296317.

Evers Rosander, E. 2003b. "Mam Diarra Bousso, the Mourid Mother in Porokhane", en Oyewumi, O. (ed.), JENdA: A Journal of Culture and African Women's Studies, Noviembre 2003.

Evers Rosander, E. 2006. "Cosmopolitas y locales. Mujeres senegalesas en movimiento", en Jabardo Velasco, M. (ed.), Senegaleses en España. Conexiones entre origen y destino: 117-129. Madrid: Ministerio de Trabajo y Asuntos Sociales.

Gemmeke, A. B. 2009. "Marabout women in Dakar: creating authority in Islamic knowledge". Africa: The Journal of the International African Institute" 79-1: 128-147.

Glover, J. 2007. Sufism and Jihad in modern Senegal. The Murid order. Nueva York: University of Rochester Press.

Glover, J. 2009. "Murid modernity. Historical perceptions of islamic reform, sufism, and colonization", en Diouf, M. y Leichtman, M. A. (eds.), New perspectives on Islam in Senegal. Conversión, migration, wealth power, and femininity: 71-90. Nueva York: Palgrave McMillan.

Gómez-Pérez, M. 1998. "Associations islamiques à Dakar", en Kane, O. y Triaud, J. L. (eds.), Islam et islamismes au sud du Sahara: 138-153. París: Karthala.

Guèye, Ch. 2008. "Touba: territorio ideal y lugar de retorno producido por los murid". Nova África 22: $59-75$.

Guèye, Ch. 2009. "Del lugar común a los 'lugares momentos', la cofradía muride y sus nuevas fronteras", en Iniesta Vernet, F. (ed.), El Islam del África negra: 91-114. Barcelona: Bellaterra.

Iniesta Vernet, F. 1998. Kuma. Historia del África negra. Barcelona: Bellaterra.

Iniesta Vernet, F. 2009. "Mil años de Islam negroafricano", en El Islam del África negra: 15-38. Barcelona: Bellaterra.

Jabardo Velasco, M. 2006. Senegaleses en España. Conexiones entre origen y destino, Madrid: Ministerio de Trabajo y Asuntos Sociales.

Jabardo Velasco, M. 2008. "Desde el feminismo negro, una mirada al género y la inmigración", en Suárez, L., Martín, E. y Hernández, R. (eds.), Feminismos en la antropología: nuevas propuestas críticas: 39-54. Donostia: Ankulegi.

Kane, O. 2011. The bomeland is the arena. Religion, transnationalism, and the integration of Senegalese immigrants in America. Oxford: Oxford University Press.

Lacomba, J. 2001. "Inmigrantes senegaleses, islam y cofradías". Revista Internacional de Sociología 29: 163-187.

Lake, R. 1997. "The making of a Mouride Mahdi: Serigne Abdoulaye Yakhine Diop of Thies", en Evers Rosander, E. y Westerlund, D. (eds.), African Islam and Islam in Africa: 216-253. Londres: Hurst \& Company.

Loimeier, R. 2009. "Dialectics of religion and politics in Senegal", en Diouf, M. y Leichtman, M. A. (eds.), New perspectives on Islam in Senegal. Conversion, migration, wealth power, and femininity: 237-256. Nueva York: Palgrave McMillan.

Mahmood, S. 2008. "Teoría feminista y el agente social dócil: algunas reflexiones sobre el renacimiento islámico en Egipto", en Suárez Navaz, L. y Hernández, R. A. (eds.), Descolonizando el feminismo. Teorías y prácticas desde los márgenes: 31-74. Valencia: Cátedra.

Massó Guijarro, E. 2010. "África en la era global: (matrias) de hospitalidad, cosmopolitismo y migración". Conferencia en el Instituto de Filosofía del CSIC, 17 de noviembre de 2010, Madrid.

Massó Guijarro, E. 2011. "La figura del marabout: ¿dominación o emancipación en la diáspora migratoria murid?". Comunicación en las VIII Jornadas Internacionales de Filosofía Política, 2124 noviembre, Universidad de Barcelona. 
Massó Guijarro, E. 2012. "Cosmopolitismo hoy: la cofradía murid y la comunidad mestiza". Intersticios. Revista Sociológica de Pensamiento Critico 6-2 (versión online, sin paginación).

Mazrui, A. 2000. "Islam between ethnicity and economic: the dialectic of Africa's experience", en Salter, T. y King, K. (eds.), Africa, Islam and development: Islam and development in Africa-African Islam, African development: 15-54. Edimburgo: University of Edinburgh.

M'Backé, K. 2005. Sufism and religious brotherhoods in Senegal. Princeton: Markus Wiener Publishers.

Moreno Maestro, S. 2007. Aqui y allí, viviendo en los dos lados. Los senegaleses de Sevilla, una comunidad transnacional. Sevilla: Junta de Andalucía.

Oyewumi, O. 2002. "Conceptualizing gender: the Eurocentric foundations of feminist concepts and the challenge of African epistemologies". JENdA: A Journal of Culture and African Women Studies 2-1.

Patterson, A. S. 1999. "The dynamic nature of citizenship and participation: lessons from three rural Senegalese case studies". Africa Today 46-1: 3-28.

Pezeril, Ch. 2008. Islam, mysticisme et marginalité: les Baay Faal du Sénégal. París: L'Harmattan.

Piga, A. 2003. L'islam in Africa. Sufismo e jibad fra storia e antropologia. Roma: Bollati.

Riccio, B. 2011. "Rehearsing Transnational Citizenship: Senegalese Associations, Co-development and Simultaneous Inclusion". African Diaspora 4: 97-113.

Rist, G. 2000. "La cultura y el capital social: ¿cómplices o víctimas del "desarrollo”?, en Klinsberg, B. y Tomassini, L. (eds.), Capital social y cultura; claves estratégicas para el desarrollo. México: Banco Iberoamericano de Desarrollo.

Roberts, A. F., Nooter Roberts, M. y Armenian, G. 2003a. "Apostle of hard work. Sheikh Ibra Fall and the Baye Fall Movement, en AA.VV (eds.), A Saint in the city: Sufi arts of urban Senegal: 109-121. Los Angeles: University of California.

Roberts, A. F., Nooter Roberts, M. y Armenian, G. 2003b. "Mouride women. In the mirror of the mother", en AA.VV (eds.), A Saint in the city: Sufi arts of urban Senegal: 151-163. Los Angeles: University of California.

Salem, G. 1981. "De la brousse sénégalaise au Boul. Mich’: le système commerciale mouride en France". Cahiers d'Études Africaines 21, 81-83: 267-88.

Salter, T. 2000. "Africa, Islam and development: three modern traditions", en Salter, T. y King, K. (eds.), Africa, Islam and development: Islam and development in Africa-African Islam, African development: 1-14. Edimburgo: University of Edinburgh.

Samson, F. 2000. "La place du religieux dans l'election présidentielle sénégalaise". Afrique Contemporaine 194: 5-11.

Samson, F. 2009. "Islam, protest, and citizen mobilization. New Sufi movements", en Diouf, M. y Leichtman, M. A. (eds.), New perspectives on Islam in Senegal. Conversión, migration, wealth power, and femininity: 257-272. Nueva York: Palgrave McMillan.

Schmitz, J. 1983. "Un politologue chez les marabouts (The Marabouts Viewed by a Political Scientist)". Cahiers d'Études Africaines 23-91: 329-351.

Shah, I. 2003. Los sufís. Barcelona: Kairós.

Skali, F. 1993. La vía sufi. España: Ibersaf Editores.

Skali, F. 2006. Jesús en la tradición sufí. España: Ibersaf Editores.

Sow, P. 2004. "Prácticas comerciales transnacionales y espacios de acción de los senegaleses en España", en Escrivà, A. y Rivas, N. (eds.), Migración y desarrollo. Estudios sobre remesas y otras prácticas transnacionales: 235-254. Córdoba: CSIC.

Suárez-Navaz, L. 2004. Rebordering the Mediterranean. Boundaries and citizenship in Southern Europe. Oxford: Berghahn Books.

Suárez Navaz, L. 2008. "La perspectiva transnacional en los estudios migratorios. Génesis, derroteros y surcos metodológicos", en García Roca, J. y Lacomba, J. (eds.), La inmigración en la sociedad española. Una radiografía multidisciplinar: 771-794. Barcelona: Bellaterra.

Talpade Mohanty, Ch. 2008a. "Bajo los ojos de Occidente": academia feminista y discursos coloniales", en Suárez-Navaz, L. y Hernández, R. A. (eds.), Descolonizando el feminismo. Teorías y prácticas desde los márgenes: 117-164. Valencia: Cátedra.

Talpade Mohanty, Ch. 2008b. "De vuelta a "Bajo los ojos de Occidente": la solidaridad feminista a través de las luchas anticapitalistas", en Suárez-Navaz, L. y Hernández, R. A. (eds.), Descolonizando el feminismo. Teorías y prácticas desde los márgenes: 407-464. Valencia: Cátedra. 
Tidiane, Ch. Sy. 1967. La confrérie sénégalaise des Mourides. París: Présence Africaine.

Vázquez Silva, I. 2010. "El régimen de cuidados en la sociedad senegalesa y sus implicaciones en el fenómeno migratorio". Comunicación en la Conferencia Anual REPS II: "Crisis económicas y politicas sociales", 30 septiembre-1 octubre 2010, Madrid.

Vázquez Silva, I. 2011. "El desafío estadístico de ser emigrante senegalés en España: un estudio comparativo de las fuentes secundarias en origen y destino sobre la inmigración senegalesa en España". Migraciones 29: 127-155.

Fecha de recepción: 22 de febrero de 2012

Fecha de aceptación: 21 de enero de 2013 